\title{
Omnimagnet: An Omnidirectional Electromagnet for Controlled Dipole-Field Generation
}

\author{
Andrew J. Petruska and Jake J. Abbott \\ Department of Mechanical Engineering, University of Utah, Salt Lake City, UT 84112 USA
}

\begin{abstract}
An Omnimagnet is an omnidirectional electromagnet comprising a spherical ferromagnetic core inside of three orthogonal nested solenoids. It generates a magnetic dipole field with both a variable dipole-moment magnitude and orientation with no moving parts. The magnetic and physical properties (e.g., dipole moment, weight, resistance, and inductance) of any Omnimagnet are derived. These general relationships are used to design an optimal Omnimagnet subject to the constraints that it has the same dipole-moment per applied current in any direction, each solenoid has no quadrupole contribution to the magnetic field, and the spherical core size maximizes the strength of the resulting dipole field. This optimal design is analyzed using FEA tools and is verified to be dipole-like in nature. Finally, the optimal design is constructed and its utility is demonstrated by driving a helical capsule-endoscope mockup through a transparent lumen.
\end{abstract}

Index Terms-Electromagnets, magnetic dipoles, magnetic manipulation, spherical core.

\section{INTRODUCTION}

$\mathbf{M}$ AGNETIC microscale and mesoscale devices (both tethered and untethered) can be manipulated with an externally generated magnetic field, which applies a combination of force and torque to the device without any mechanical connection. Although a combination of permanent magnets and electromagnets can be used to produce the magnetic field required for a manipulation task, some tasks seem better suited to either permanent-magnet or electromagnet systems. Because they have more direct real-time control of the applied magnetic field, electromagnet systems have been used for multidegreeof-freedom levitation and position/orientation control [1]-[8]. Permanent magnets, which require no electrical power to generate a strong field, have been used for pulling and rolling tasks in which the environment provides some structure [9]-[12], as well as for quasi-static pointing tasks of tethered devices, such as magnetic catheters [13]. Because both attractive and lateral forces can be generated between a rotating dipole source and a sympathetically rotating magnetic device, a rotating dipole field could be more effective for rolling/screwing propulsion than the rotating uniform field generated by many electromagnet systems [14]. Finally, it is challenging to scale many laboratory electromagnetic systems that surround their workspace (e.g., Helmholtz coils) to a size that would be required for medical applications, whereas manipulation systems that utilize dipole fields can be located adjacent to their workspace.

An omnidirectional electromagnet, formed by any set of collocated electromagnets that have dipole moments spanning $\mathbb{R}^{3}$, combines the real-time control of field strength associated with traditional electromagnets and the control of dipole orientation associated with rotating permanent magnets. In this paper, we describe the Omnimagnet, a subclass of

Manuscript received June 19, 2013; accepted January 8, 2014. Date of publication January 30, 2014; date of current version July 7, 2014. Corresponding author: A. J. Petruska (e-mail: andrew.petruska@utah.edu).

Color versions of one or more of the figures in this paper are available online at http://ieeexplore.iee.org.

Digital Object Identifier 10.1109/TMAG.2014.2303784

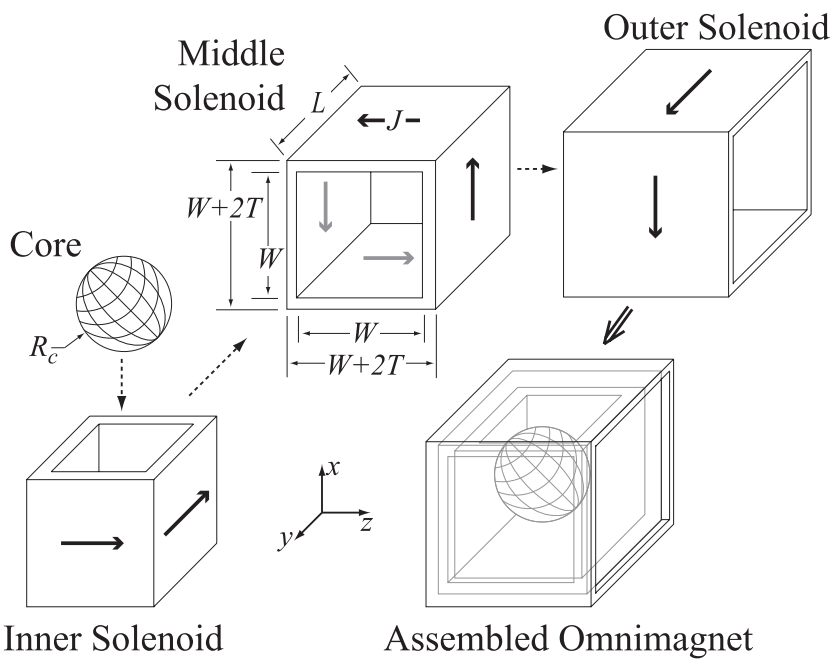

Fig. 1. Assembled Omnimagnet forms a cube constructed of three nested orthogonal solenoids surrounding a spherical core of ferromagnetic material. Each square-cross section solenoid has a different inner width $W$, winding thickness $T$, length $L$, and associated current density $J$.

omnidirectional electromagnets that comprise a ferromagnetic core surrounded by three orthogonal solenoids. Specifically, we optimize an Omnimagnet comprising a spherical core and square-cross section solenoids (Fig. 1), but other design variations could be considered. An Omnimagnet creates a fully controllable dipole-like magnetic field, contains no moving parts, and becomes inert when powered down-reducing the safety concerns associated with permanent-magnet field sources. The concept of three nested solenoids has been explored as a method of magnetic localization [15] and inductive power coupling [16], but never with a spherical core, and never as a dipole-like magnetic manipulator.

This paper is structured as follows. First, the general design problem for an Omnimagnet is presented. Next, the magnetic fields generated by the three solenoids are described using a multipole expansion of the magnetostatic equations, and the contribution of the ferromagnetic core is quantified. The optimization of a specific Omnimagnet follows, and the 
design is described. The field generated by this design is then compared with a dipole-field approximation. The inverse solution for determining the dipole moment, and thus the currents, required to produce a desired static or rotating field at a given location is provided. Finally, the capability of the Omnimagnet for the control of untethered magnetic devices is then demonstrated by driving a mockup capsule endoscope through a lumen. This paper expands on our result originally presented in [17] by providing: a more detailed treatment of the quadruple minimization, methods for calculating other relevant mechanical, electrical, and magnetic properties, and an analysis of how extraneous magnetic fields will affect the core magnetization.

\section{Design AND Optimization}

The general concept of an Omnimagnet is broad, consisting of three orthogonal nested solenoids surrounding a ferromagnetic core; however, design choices must be made to realize and optimize a physical Omnimagnet. First, we chose the shape of the solenoids to be square-cross sectional sleeves to result in a dense packing (Fig. 1). Next, we chose the core to be a sphere because a spherical core has three desirable properties [18].

1) A sphere does not have a preferential magnetization direction.

2) When placed in a uniform field (similar to the field in the center of a solenoid), a sphere produces a pure dipole field.

3) The average applied magnetic field within a sphere is equal to the applied magnetic field at the center of the sphere.

We chose that the dipole-moment generated in each direction, which consists of the contribution of both an individual solenoid and the magnetization of the core due to that solenoid, should be the same when an equal electrical current density is applied through each solenoid. Other geometric design choices (e.g., cylindrical solenoids or a cubic core) or dipolemoment relationships (e.g., scaling the dipole moment of each solenoid with its heat-transfer capability), could also be pursued using the general framework for Omnimagnet design outlined below. Finally, we constrain our design to use a single wire gauge for all solenoids, which means that an equal electrical current density is synonymous with an equal current; current and current density are related by the cross sectional area of the wire used. Throughout this paper, $I$ will be used to refer to currents in units $\{\mathrm{A}\}$ and $J$ will be used to refer to current density in units $\left\{A \cdot m^{-2}\right\}$. Because current density is invariant to wire selection, the optimization for shape is performed using $J$; general discussion, however, will use $I$, as it is the more natural parameter from a control perspective. The final design of the Omnimagnet shown in Fig. 1 requires ten total constraints (the length, width, and thickness of each solenoid, and the radius of the core).

The magnetic field generated by the Omnimagnet can be represented by the field contributed by the magnetized spherical core superimposed with the field contributed by the solenoids. Modeling the total field can be performed using FEA tools with a resolution limited by the number of elements used. Alternatively, an analytical dipole approximation can be used to model the field. The dipole approximation provides a closed-form vector equation that can be used to calculate the field generated at a point, or inverted to determine the current necessary to create a particular field. The closer the Omnimagnet is to generating a pure dipole field, the better the algorithms based on this approximation will perform [12], [14], [15], [19], [20]. By correctly choosing the solenoids' aspect ratios, the dipole-approximation error can be minimized as a part of the design optimization.

\section{A. Solenoid Multipole Field Expansion}

For positions outside of the Omnimagnet's minimumbounding sphere (i.e., the smallest sphere that the Omnimagnet can fit within), the solenoids' fields can be represented by a multipole expansion of a vector potential [18]

$$
\begin{aligned}
& \mathbf{B}(\mathbf{p})=\nabla \times \Psi(\mathbf{p}) \\
& \Psi(\mathbf{p})=\frac{\mu_{0}}{4 \pi} \sum_{n=0}^{\infty} \frac{1}{\|\mathbf{p}\|^{n+1}} \int_{\mathcal{V}_{s}} \mathbf{J}(\mathbf{r})\|\mathbf{r}\|^{n} P_{n}(\hat{\mathbf{p}} \cdot \hat{\mathbf{r}}) d V
\end{aligned}
$$

where $\mu_{0}=4 \pi \times 10^{-7} \mathrm{~T} \cdot \mathrm{m} \cdot \mathrm{A}^{-1}$ is the magnetic permeability of free space, $\mathbf{p}$ is the vector (with associated unit vector $\hat{\mathbf{p}}$ ) from the center of the Omnimagnet to the point of interest in units $\{\mathrm{m}\}, \mathbf{r}$ is the vector (with associated unit vector $\hat{\mathbf{r}}$ ) from the center of the Omnimagnet to the point in the solenoid being integrated, $\mathbf{J}(\mathbf{r})$ is the current density vector that points in the direction of the current flow at location $\mathbf{r}, \mathcal{V}_{s}$ represents the solenoid's volume, and $P_{n}()$ are the Legender polynomials. Since the divergence of a magnetic field through a closed surface must be zero, all of the even terms (those corresponding to $\left.P_{0}, P_{2}, \ldots\right)$ must be zero, leaving only the odd terms. The first nonzero term in the multipole expansion (corresponding to $\left.P_{1}\right)$ is the dipole field, which can be expressed in a coordinate-free form

$$
\mathbf{B}(\mathbf{p})=\frac{\mu_{0}}{4 \pi\|\mathbf{p}\|^{3}}\left(3 \hat{\mathbf{p}} \hat{\mathbf{p}}^{\mathrm{T}}-\mathbb{I}\right) \mathbf{m}
$$

where $\mathbb{I}$ is a $3 \times 3$ identity matrix and $\mathbf{m}$ is the dipole moment in units $\left\{A \cdot m^{2}\right\}$. The dipole moment for a current density of any configuration is [18]

$$
\mathbf{m}=\frac{1}{2} \int_{\mathcal{V}_{s}} \mathbf{r} \times \mathbf{J}(\mathbf{r}) d V .
$$

The dipole moment for a square-cross section solenoid, as shown in Fig. 1 with uniform current density (i.e., the current density does not vary along the thickness or length of the solenoid) is

$$
\mathbf{m}=\frac{J L^{4}}{6}\left(\beta_{2}^{3}-\beta_{1}^{3}\right) \hat{\mathbf{l}}
$$

where $J=\|\mathbf{J}\|, L$ is the axial length of the solenoid (with associated axial unit vector $\hat{\mathbf{l}}$ ), and $\beta_{1}=W / L$ and $\beta_{2}=$ $(W+2 T) / L$, respectively, describe the inner-width-to-length and outer-width-to-length aspect ratios. 
The maximum dipole moment that any electromagnet with a bounding cube of edge length $L$ containing no ferromagnetic material could generate in one direction can be calculated by (5) with $\beta_{1}=0$ and $\beta_{2}=1$, and is $J L^{4} / 6$. The maximum theoretical dipole moment that could be expected for any cubic omnidirectional electromagnet with edge length $L$ containing no ferromagnetic material is thus $1 / 3$ of the unidirectional case: $J L^{4} / 18$; this quantity is used throughout this paper to normalize the strength for a nondimensional optimization, although constructing such an idealized omnidirectional electromagnet would be very challenging.

We show in [21] that by varying the aspect ratios of a rectangular permanent magnet, the dipole-field approximation error can be minimized. The approach finds the geometry that sets the next term in the multipole expansion, the quadrupole term, to zero in the scalar potential of the magnetic field. Using the same technique, but using the vector potential instead of a scalar potential, the magnetic field of each solenoid can be shaped to produce a dipole-like field by removing the quadrupole contribution to the multipole expansion. The quadrupole term for a square-cross section solenoid of uniform current density corresponds to the $P_{3}$ term in the expansion

$$
\begin{aligned}
\mathbf{B}_{\text {quad }}(\mathbf{p})= & \frac{\mu_{0}}{4 \pi} \frac{1}{\|\mathbf{p}\|^{5}}\left(\left(35\left(\hat{\mathbf{m}}^{\mathrm{T}} \hat{\mathbf{p}}\right)^{2}-15\right) \hat{\mathbf{p}} \hat{\mathbf{p}}^{\mathrm{T}}\right. \\
& \left.-\left(15\left(\hat{\mathbf{m}}^{\mathrm{T}} \hat{\mathbf{p}}\right)^{2}-3\right) \mathbb{I}\right) \mathbf{m}_{\text {quad }}
\end{aligned}
$$

where $\mathbf{m}_{\text {quad }}$ is the quadrupole moment, given by

$$
\mathbf{m}_{\text {quad }}=\frac{3}{40} L^{2}\left(\beta_{1}^{2}+\frac{\beta_{2}^{4}+\beta_{1} \beta_{2}^{3}}{\beta_{1}^{2}+\beta_{1} \beta_{2}+\beta_{2}^{2}}-\frac{5}{3}\right) \mathbf{m} .
$$

The values of $\beta_{1}$ and $\beta_{2}$ that set (7) to zero correspond to geometries with minimal dipole-field approximation error. Letting $\beta_{1}=\alpha \beta_{2}$ and substituting into the polynomial in (7) and setting to zero gives an alternate equation for zero quadruple moments

$$
3 \beta_{2}^{4}\left(\alpha^{4}+\alpha^{3}+\alpha^{2}+\alpha+1\right)-5 \beta_{2}^{2}\left(\alpha^{2}+\alpha+1\right)=0 .
$$

Inspection of (8) shows there is no quadrupole moment when $\beta_{1}=\beta_{2}=0$ (no magnet), when $\beta_{1}=\beta_{2}=\alpha=1$ (thin walled shell), and when $\beta_{1}=0, \beta_{2}=\sqrt{5 / 3}, \alpha=0$ (no inner hole). As the definition of $\beta_{1}$ and $\beta_{2}$ requires $\beta_{1} \leq \beta_{2}$, the only physically meaningful solutions to (8) lie in the range $\beta_{1} \in(0,1)$ and $\beta_{2} \in(1, \sqrt{5 / 3})$, which correspond to geometries that are shorter than they are wide $(L<W+2 T)$. A fit of the roots of (8) can be used to provide an approximation of the relationship between $\beta_{1}$ and $\beta_{2}$

$$
\begin{aligned}
\beta_{2} & \approx\left((5 / 3)^{n / 2}-\beta_{1}^{n}\right)^{1 / n} \quad\left(0 \leq \beta_{1} \leq 1\right) \\
n & =\frac{2 \log 2}{\log (5 / 3)} \approx 2.714
\end{aligned}
$$

which provides solutions to (8) accurate to within $0.17 \%$ over the range $\beta_{1} \in(0,1)$.

\section{B. Core Dipole-Field Contribution}

Since the core is spherical and placed in the nearly uniform field inside of the solenoid, it is assumed that it will magnetize uniformly and contribute a pure dipole field (it was verified numerically post facto that the root-mean-squared deviation in the magnetization was less than $7 \%$ of the mean).

The dipole moment of a low coercivity, low remanence, and high permeability $(\chi \gg 1)$ spherical core, when magnetized in its linear region (i.e., below magnetic saturation), is

$$
\mathbf{m}_{c}=\overline{\mathbf{M}} \mathcal{V}_{c}=\left(\frac{\chi}{1+\frac{1}{3} \chi} \frac{\overline{\mathbf{B}}}{\mu_{0}}\right)\left(\frac{4 \pi}{3} R_{c}^{3}\right) \approx \frac{4 \pi R_{c}^{3}}{\mu_{0}} \mathbf{B}_{c}
$$

where $R_{c}$ is the radius of the core, $\mathbf{M}$ is the magnetization in units $\left\{\mathrm{A} \cdot \mathrm{m}^{-1}\right\}$, the overbar represents a quantity averaged over the core volume $\mathcal{V}_{c}$, and $\mathbf{B}_{c}$ is the applied magnetic field at the center of the core, which is a linear combination of the field due to each solenoid. The applied field from each square-cross section solenoid with uniform current density $J$, length $L$, and axis $\hat{\mathbf{i}}$, calculated by the Biot-Savart law, is

$$
\mathbf{B}_{c}=\frac{2 L J \mu_{0}}{\pi} \int_{\beta_{1}}^{\beta_{2}} \operatorname{atan}\left(\frac{1}{\sqrt{1+2 \zeta^{2}}}\right) d \zeta \hat{\mathbf{l}} .
$$

By combining the dipole moments due to the magnetized core and each of the solenoids, the total dipole moment of the Omnimagnet $\mathbf{m}=\mathbf{m}_{x}+\mathbf{m}_{y}+\mathbf{m}_{z}$ is thus

$$
\begin{gathered}
\mathbf{m}=\sum_{i \in\{x, y, z\}} J_{i}\left(8 L_{i} R_{c}^{3} \int_{\beta_{i, 1}}^{\beta_{i, 2}} \operatorname{atan}\left(\frac{1}{\sqrt{1+2 \zeta^{2}}}\right) d \zeta\right. \\
\left.+\frac{L_{i}^{4}}{6}\left(\beta_{i, 2}^{3}-\beta_{i, 1}^{3}\right)\right) \hat{\mathbf{l}}_{i} \\
=\mathbb{M}\left[\begin{array}{l}
J_{x} A_{w, x} \\
J_{y} A_{w, y} \\
J_{z} A_{w, z}
\end{array}\right]=\mathbb{M I}
\end{gathered}
$$

where the indices $x, y$, and $z$ correspond to the inner, middle, and outer solenoids, respectively, and without loss of generality, $A_{w}$ is the conductor (e.g., wire) area used to wind the solenoid, and $\mathbb{M}$ is a linear transformation that maps the three applied currents in the array $\mathbf{I}$ to the dipole moment $\mathbf{m}$. Since the matrix $\mathbb{M}$ is only a function of the solenoids' geometries, the optimization problem can be split into four steps: 1) choose the maximum current densities $\mathbf{I}_{\max }$ in each direction based on thermal or amplifier constraints; 2) determine the geometric $\beta$ factors to equalize the components of $\mathbb{M} \mathbf{I}_{\max }$; 3) optimize the overall size to a set of physical and operational constraints (e.g., field strength, field gradient, weight, and electrical time constant); and 4) finally, tailor the wire gage for manufacturability or amplifier voltage/current limitations.

\section{Dipole-Moment Equalization}

The optimal geometry for the Omnimagnet corresponds to the geometric ratios that maximize the dipole-moment generated in each direction, have the same ratio of dipole moment to maximum current density in each direction, and have no quadruple moment. This is a constrained optimization problem, and can be nondimensionalized by dividing all of the lengths by $L_{\max }$ (the edge length of a minimum-bounding cube) and the moments by $m_{\text {ref }}=J_{\max } L_{\text {max }}^{4} / 18$ (the maximum 


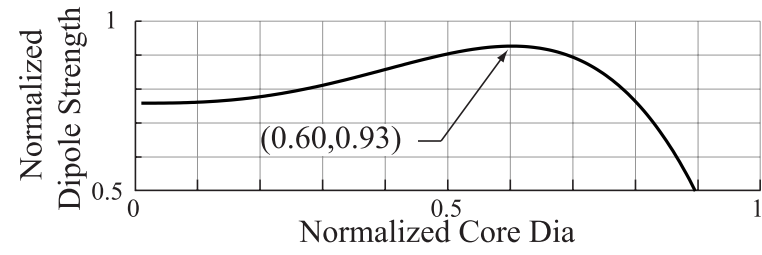

\begin{tabular}{|c|c|c|c|c|c|}
\hline & $L / L_{\max }$ & $W / L_{\max }$ & $T / L_{\max }$ & $\beta_{1}$ & $\beta_{2}$ \\
\hline $\mathrm{x}$ & 0.70 & 0.60 & 0.09 & 0.86 & 1.11 \\
\hline $\mathrm{y}$ & 0.84 & 0.78 & 0.06 & 0.92 & 1.07 \\
\hline $\mathrm{z}$ & 0.96 & 0.91 & 0.05 & 0.95 & 1.05 \\
\hline
\end{tabular}

Fig. 2. Optimal geometry for a no-quadrupole Omnimagnet. The dipole moments have been normalized by $J L_{\max }^{4} / 18$ and have a maximum at a core-diameter-to-outer-Omnimagnet-dimension ratio $2 R_{c} / L_{\max }=0.60$. The table provides the geometric ratios that describe the shape of the three nested solenoids that correspond to this optimal configuration. All length parameters are normalized by the outer Omnimagnet cubic length $L_{\max }$.

no-ferromagnetic-material dipole moment introduced earlier). The constraints can be simplified because, from (7), the length of each solenoid must be shorter than its outer width. Thus, the objective is to maximize the dipole moment magnitude $\|\mathbf{m}\|$, which is subjected to the following.

1) Equation (7) equals zero (i.e., the configuration has no quadrupole moment).

2) $\left\|\mathbf{m}_{x}\right\| / m_{\text {ref }}=\left\|\mathbf{m}_{y}\right\| / m_{\text {ref }}=\left\|\mathbf{m}_{z}\right\| / m_{\text {ref }}$ (i.e., omnidirectionality).

3) $W_{x}=2 R_{c}$ (i.e., the core diameter is the same as the inner solenoid's inner width).

4) $W_{y}=W_{x}+2 T_{x}$ (i.e., the inner solenoid's outer width is the same size as the middle solenoid's inner width).

5) $W_{z}=W_{y}+2 T_{y}$ (i.e., the middle solenoid's outer width is the same size as the outer solenoid's inner width).

This optimization is performed using $R_{c}$ as the free parameter (Fig. 2). There is a maximum that occurs when the core diameter is $60 \%$ of $L_{\max }$. Although the magnitude of the dipole moment in each direction is the same for the same applied current, the percentages of the dipole-moment attributed to the core or the windings are different for each solenoid; the percentage of the dipole moment from the core/windings is $41 / 59,28 / 72$, and $21 / 79$ for the inner, middle, and outer solenoids, respectively. The optimized configuration has dipole-moment magnitudes that are $93 \%$ of what could be theoretically expected with no ferromagnetic material and no voids (an unrealizable geometry), and $22 \%$ greater than the realizable geometry of three nested solenoids with no ferromagnetic core (but with significantly less power consumption and more heat-transfer surface area). The optimal design's dipole moment is

$$
\begin{aligned}
\mathbf{m} & =61.86 \times 10^{-3} L_{z}^{4} \mathbf{J} \\
& =51.45 \times 10^{-3} L_{\max }^{4} \mathbf{J} .
\end{aligned}
$$

Solutions to the right of the maximum in Fig. 2 correspond to geometries with more inert (i.e., noncurrent-energized) material and will produce less heat and require less power than the corresponding geometry to the left of the optimal point. The flatness of the maximum indicates that variations about the optimal point will not substantially affect the performance
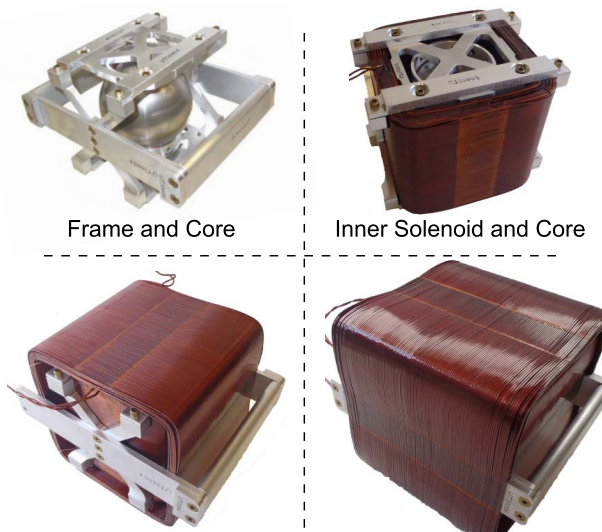

Middle and Inner Solenoid

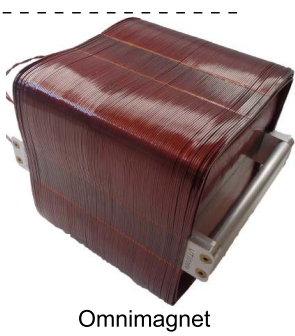

\begin{tabular}{|c|c|c|c|c|c|}
\hline & $L(\mathrm{~mm})$ & $W(\mathrm{~mm})$ & $T(\mathrm{~mm})$ & $\beta_{1}$ & $\beta_{2}$ \\
\hline $\mathrm{x}$ & 120 & 103 & 16 & 0.86 & 1.12 \\
\hline $\mathrm{y}$ & 149 & 137 & 11 & 0.92 & 1.07 \\
\hline $\mathrm{z}$ & 170 & 160 & 8 & 0.95 & 1.05 \\
\hline
\end{tabular}

Fig. 3. Assembled Omnimagnet used in the testing described in this paper. The core used is $100 \mathrm{~mm}$, which is $57 \%$ of $L_{\max }=176 \mathrm{~mm}$. The deviations from Fig. 2 are due to reoptimizing with $1 \mathrm{~mm}$ airgaps between the components for assembly.

of the resulting Omnimagnet. Fortunately, the solenoids are shorter than they are wide, which allows paths for conductors and coolant to reach the middle and inner solenoids, making the implementation of this design feasible.

\section{Prototype}

An Omnimagnet was constructed using a $100 \mathrm{~mm}$ diameter spherical Nickel-Iron (ASTM A753-08-K94840) core. The core material was chosen because it has a high magnetic permeability, a low magnetic remanence, and a low magnetic coercivity (i.e., it magnetizes easily but does not remain magnetized when the magnetizing field is removed). The solenoids were constructed using 16 AWG square self-bonding copper wire from MWS Wire Industries, Inc. The optimization was repeated including a $1 \mathrm{~mm}$ spacing between each of the components for assembly. The slightly modified optimization did not change the shape of the coils substantially but shifted the optimal core size to $57 \%$ of the edge length of the minimum-bounding cube, which is $L_{\max }=176 \mathrm{~mm}$. The additional empty space also reduced the overall strength of the design from $93 \%$ to $87 \%$ of $m_{\text {ref. }}$. Because of the quantization in lengths and widths inherent with any winding, the constructed Omnimagnet has slight variations in the dipole-moment strengths of each solenoid and has successfully minimized, but not eliminated, the quadrupole term $\left(\mathbf{m}_{\text {quad }} \approx 0.04 \mathbf{m}\right)$. The prototype constructed is shown in Fig. 3, with the table providing its dimensions; the dipolemoment per conductor-current is calculated to be 25.1, 25.8 , and $26.3\left(\mathrm{~A} \cdot \mathrm{m}^{2}\right) / \mathrm{A}$ for the inner, middle, and outer solenoids, respectively, which form the diagonal entries of $\mathbb{M}$. The field at the surface with $1 \mathrm{~A}$ applied is measured to be 5.6, 4.7, and $3.6 \mathrm{mT}$ for the inner, middle, and outer solenoids, respectively; at $12 \mathrm{~cm}$ from the surface the field is $0.6 \mathrm{mT}$ 

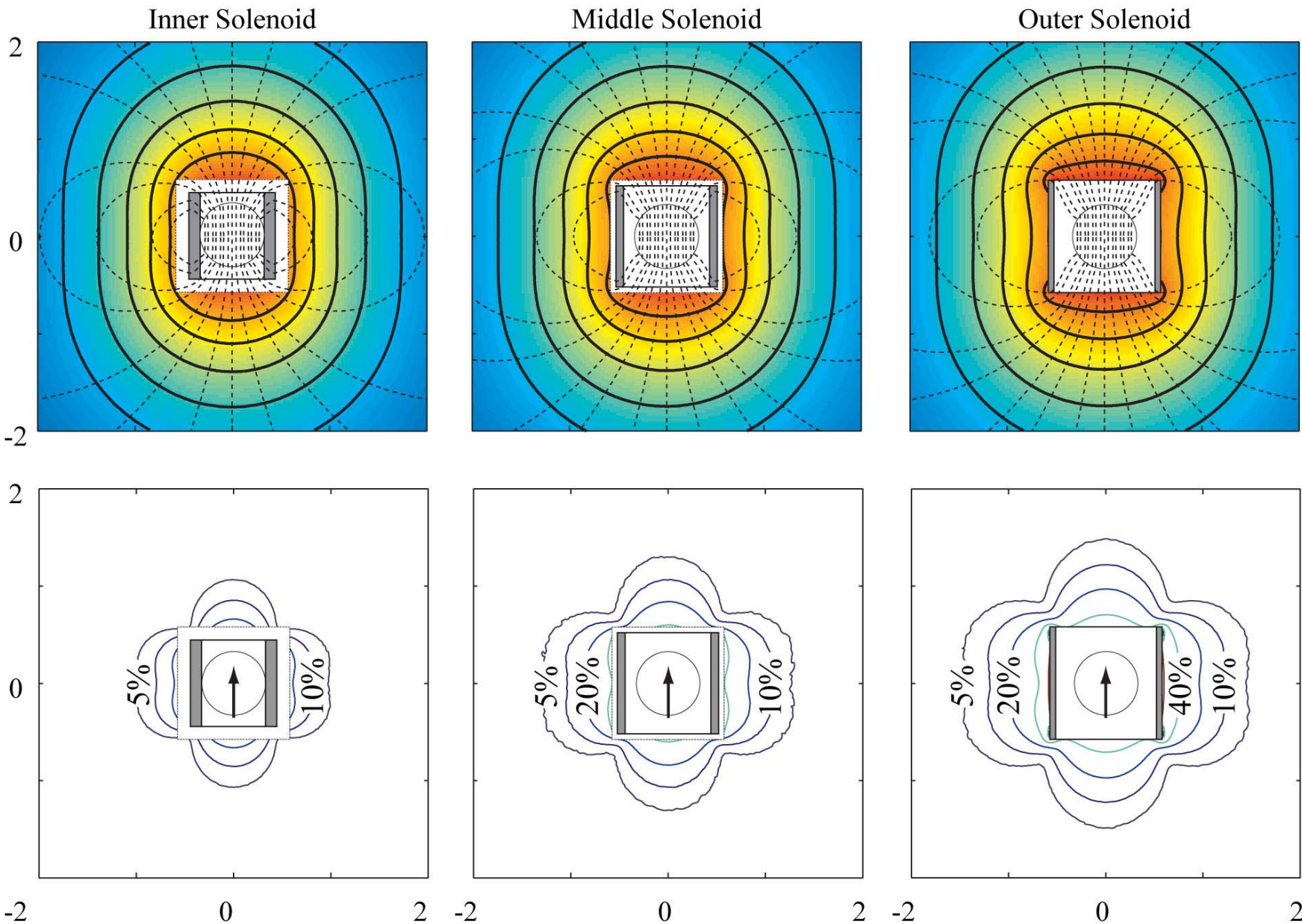

Fig. 4. Field shape (top, dotted) and magnitude (top, solid and color contour), and field error relative to the dipole approximation (bottom) are shown. The distances are normalized by the radius of a minimum-bounding sphere: $(\sqrt{3} / 2) L_{\max }$. In the top row, the innermost contour line corresponds to $\|\mathbf{B}\|=64 \mu_{0}\|\mathbf{m}\|$, and each successively larger contour corresponds to a halving in field magnitude, with the outermost contour corresponding to $\|\mathbf{B}\|=4 \mu_{0}\|\mathbf{m}\|$.

for each. The inductance for each solenoid in the assembled Omnimagnet was measured to be 120,107 , and $78 \mathrm{mH}$ for the inner, middle, and outer solenoids, respectively.

Since each solenoid in the Omnimagnet has a different geometry, the magnetic field produced by each solenoid will not have exactly the same shape for positions close to the Omnimagnet. To understand the subtle differences in field shape, FEA simulations were performed using Ansoft Maxwell 14.0. Since the core is magnetized in the linear region and the solenoids are orthogonal, solenoid-solenoid magnetic coupling is negligible, so in these simulations only one of the solenoids is energized at a time. The results of the simulation (field strength, field shape, and percent error from the pointdipole approximation) for each solenoid are shown in Fig. 4. As the outermost solenoid is the largest, it is responsible for the majority of the field deviations close to the Omnimagnet. The field in each direction rapidly reduces to a pure dipole field with distance; the deviations are comparable to non-spherical permanent magnets [21].

\section{EfFect of Adjacent Magnetic Sources}

Because the Omnimagnet contains a spherical ferromagnetic core, any adjacent magnetic source (e.g., a permanent magnet used in a magnetic tool) will slightly magnetize the core, causing the resulting dipole moment of the Omnimagnet to be perturbed. The effect of the external field on the core is given by (10) where $\mathbf{B}_{c}=\mathbf{B}_{c, p}$ is given by the value of the external field that is perturbing the magnetization at the center of the core. Due to the linearity of the magnetics equations when the core is unsaturated, the resulting Omnimagnet dipole moment is the sum of the original desired moment and the perturbed moment

$$
\mathbf{m}=\mathbb{M I}+\frac{4 \pi R_{c}^{3}}{\mu_{0}} \mathbf{B}_{c, p} .
$$

To account for perturbing field sources, it is necessary to subtract their effect from the desired dipole moment prior to calculating the currents required.

\section{Physical Properties}

The primary design of an Omnimagnet is focused on the optimization of its magnetic properties. However, any physical system will have to be designed around other limitations, such as weight, electrical resistance, electrical inductance, and heating limits. 


\section{A. Weight}

The weight of the Omnimagnet is purely a function of volume

$$
W=\rho_{\text {core }} \frac{4 \pi R_{c}^{3}}{3}+\rho_{s} \sum_{i \in\{x, y, z\}} L_{i}^{3}\left(\beta_{i, 2}^{2}-\beta_{i, 1}^{2}\right)
$$

where $\rho_{\text {core }}$ and $\rho_{s}$ are the densities of the core and solenoid material, respectively, in units $\left(\mathrm{kg} \cdot \mathrm{m}^{-3}\right)$.

\section{B. Resistance and Heating/Cooling Limits}

The electrical resistance of each solenoid is a function of both the volume and the wire size chosen

$$
R=\frac{\rho_{w} \eta L^{3}}{A_{w}^{2}}\left(\beta_{2}^{2}-\beta_{1}^{2}\right)
$$

where $\rho_{w}$ is the resistivity of the wire in units $(\Omega \cdot \mathrm{m}), A_{w}$ is the cross-sectional area of the conductor used, and $\eta$ describes the packing density of the conductor ( 1 for square wire and $\pi / 4$ for round wire). The resistance of the Omnimagnet system can be described by the diagonal matrix $\mathbb{R}$, which is formed by packing each coil's resistance along the diagonal. With this definition the power lost to Joule heating becomes

$$
\dot{q}_{\text {gen }}=\mathbf{I}^{\mathrm{T}} \mathbb{R} \mathbf{I} .
$$

For the Omnimagnet to be operating at thermal equilibrium, the heat generated $\dot{q}_{\text {i,gen }}$ must be balanced by the heat conducted or convected from the coil to the environment via a fluid (e.g., air, transformer oil). The heat transfer rate is a function of surface area and temperature difference

$$
\dot{q}_{i, \text { out }}=2 h_{i} L_{i}^{2}\left(\beta_{i, 2}^{2}-\beta_{i, 1}^{2}+2\left(\beta_{i, 2}+\beta_{i, 1}\right)\right) \Delta T_{i}
$$

where $h$ describes the total heat transfer coefficient and $\Delta T$ is the temperature difference between the coil and the environment. The equilibrium temperature is reached when the heat generated by Joule heating is balanced by the heat convected out of the system, which determines the maximum current density

$$
J_{i, \max }=\sqrt{\frac{h_{i} \Delta T_{i}}{L_{\max } \rho_{w}} \frac{2\left(\beta_{i, 2}^{2}-\beta_{i, 1}^{2}+2\left(\beta_{i, 2}+\beta_{i, 1}\right)\right)}{\left(L_{i} / L_{\max }\right)\left(\beta_{i, 2}^{2}-\beta_{i, 1}^{2}\right)}} .
$$

From the definition of the Nusselt number and assuming forced convection in a channel, we have $h \propto L^{-1}$, which implies $J_{\max } \propto L_{\max }^{-1}$. The maximum dipole moment that can be generated is thus

$$
\mathbf{m}_{\max }=\mathbb{M} \mathbf{J}_{\max } \propto L_{\max }^{3}
$$

which is the same scaling as would be expected for a permanent magnet. The magnetic field generated scales homothetically and the field gradients, which are proportional to the force applied to an adjacent magnet, scale inversely with $L_{\max }$.

\section{Inductance and Time Constant}

The inductances, and therefore time constants, of the Omnimanget solenoids are difficult to calculate exactly because of their shape and the presence of the spherical core. However, an approximation can be derived that will suffice for use in Omnimagnet design. The approach separates the problem into two parts: the inherent inductance in the solenoid due to the windings, and the additional inductance due to the spherical core. As shown in the derivation provided in the Appendix, the inductance $\mathcal{L}$ can be written as a function of relative solenoid shape scaled by a function of solenoid size

$$
\begin{aligned}
\mathcal{L} \approx & \frac{\mu_{0} \eta L^{5}}{6 \pi A_{w}^{2}} f_{1}\left(\beta_{1}, \beta_{2}, \beta_{c}\right) \\
f_{1}\left(\beta_{1}, \beta_{2}, \beta_{c}\right)= & \left(\beta_{2}^{3}-\beta_{1}^{3}\right) \int_{-1 / 2}^{1 / 2} \int_{\beta_{1}}^{\beta_{2}} f_{2}\left(\zeta_{1}, \zeta_{2}\right) d \zeta_{1} d \zeta_{2} \\
& +\pi \beta_{c}^{3}\left(\beta_{2}-\beta_{1}\right) \int_{\beta_{1}}^{\beta_{2}} f_{2}\left(\zeta_{1}, 0\right) d \zeta_{1} \\
f_{2}\left(\zeta_{1}, \zeta_{2}\right)= & \arctan \left(\frac{1+2 \zeta_{2}}{\sqrt{\left(1+2 \zeta_{2}\right)^{2}+2 \zeta_{1}^{2}}}\right) \\
& +\arctan \left(\frac{1-2 \zeta_{2}}{\sqrt{\left(1-2 \zeta_{2}\right)^{2}+2 \zeta_{1}^{2}}}\right)
\end{aligned}
$$

where $\beta_{c}$ is the ratio of core diameter to solenoid length. The calculated inductances for the as-built configuration are 163, 110, and $82 \mathrm{mH}$, for the inner, middle, and outer solenoids, respectively. This is within $35 \%$ of the measured values reported above, indicating reasonable agreement between the approximation and the measurement. The values of $f_{1}$ for the optimal geometry defined earlier are 0.372 , 0.089 , and 0.037 for the inner, middle, and outer solenoids, respectively.

The time constant $\tau$ for each solenoid is

$$
\tau=\frac{\mathcal{L}}{R} \approx L^{2} \frac{\mu_{0}}{2 \pi \rho_{w}} \frac{f_{1}\left(\beta_{1}, \beta_{2}, \beta_{c}\right)}{\beta_{2}^{2}-\beta_{1}^{2}}
$$

Although the system resistance and inductance can be tailored through wire selection, the time constant is independent of wire choice. Since the equations for time constant and dipole moment are functions of the same variables, it is not possible to design the two properties independently.

\section{Field Control}

Fundamentally, the Omnimagnet is a magnetic field source with three control inputs (the current applied to each solenoid), which can be used to generate a desired magnetic field at a location in space. Since the magnetic field generated is closely represented by a dipole field, at each location in space it will produce both a field and spatial derivatives in the field. With a single Omnimagnet with $3 \mathrm{DoF}$, it is not possible to independently control field and field gradient simultaneously.

To determine the dipole moment $\mathbf{m}$ required by the Omnimagnet to produce a desired field $\mathbf{B}$ at some point $\mathbf{p}$, 

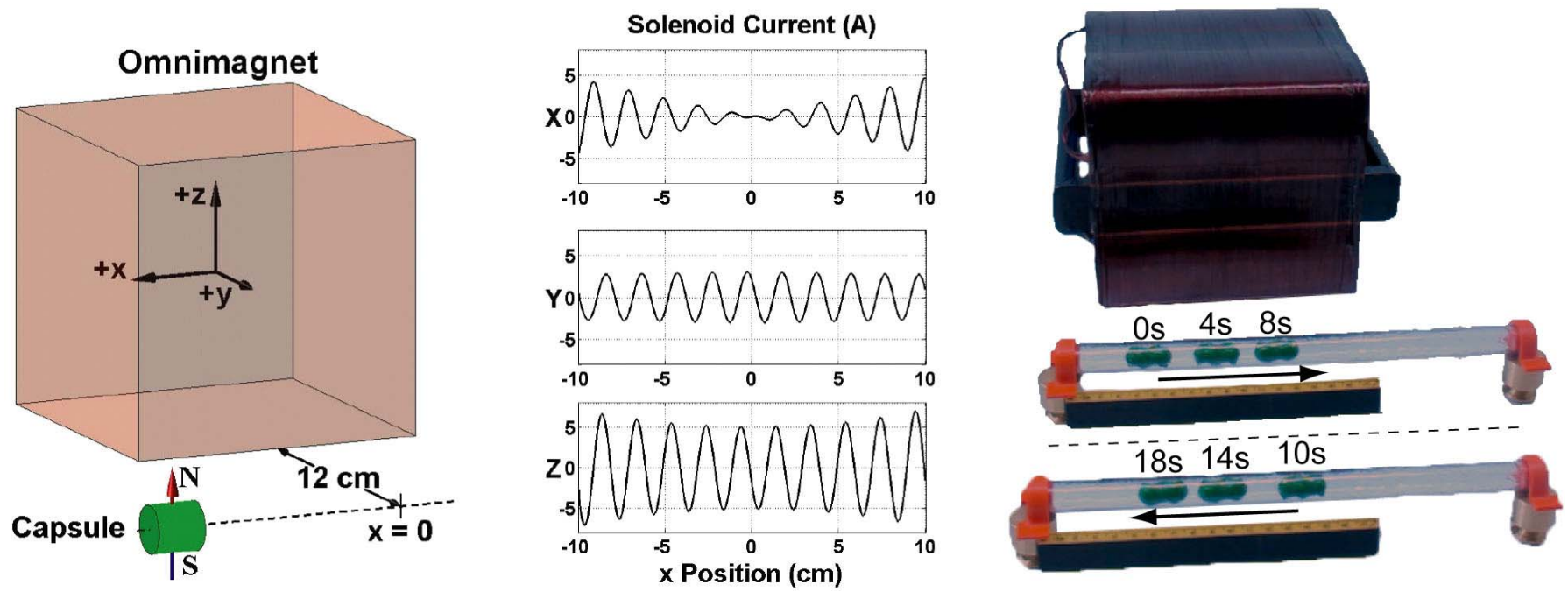

Fig. 5. Propulsion of a helical capsule at $10 \mathrm{~mm} \cdot \mathrm{s}^{-1}$ through a lumen located $120 \mathrm{~mm}$ from the surface of the Omnimagnet, which is applying a rotating magnetic field at the location of the helical capsule. Left: Numerical simulation with resulting solenoid currents (center). Right: Experimental demonstration with a capsule mockup.

the vector dipole equation (3) must be inverted. Using the dipole field analysis in [12], it can be shown that the inverse exists of the form

$$
\mathbf{m}=\frac{2 \pi}{\mu_{0}}\|\mathbf{p}\|^{3}\left(3 \hat{\mathbf{p}} \hat{\mathbf{p}}^{\mathrm{T}}-2 \mathbb{I}\right) \mathbf{B} .
$$

Combining with (12), the currents required are thus

$$
\mathbf{I}=\frac{2 \pi}{\mu_{0}}\|\mathbf{p}\|^{3} \mathbb{M}^{-1}\left(3 \hat{\mathbf{p}} \hat{\mathbf{p}}^{\mathrm{T}}-2 \mathbb{I}\right) \mathbf{B} .
$$

\section{Vi. Remote Manipulation With an OMNimagnet}

At steady state, the torque applied by a magnetic field on a magnetic tool will tend to align the tool with the field. This can be used to reduce the control problem from controlling torque directly, and thus requiring both tool orientation and position, to controlling field directly, and thus requiring only tool position. Controlling the propulsion of a ball or helical screw with this approach has been explored using a rotating permanent magnet [12]. However, when a permanent magnet is used to create a rotating field, both the magnitude and rotation rate of the field vary elliptically at the tool location; thus, the rotational speed of the permanent magnet needs to be constantly updated to produce a constant tool rotation rate [12]. Since the Omnimagnet can produce a desired field at a specific point in space, it is possible to create a rotating field with angular velocity $\omega$ without the elliptical modulation in magnitude associated with rotating permanent magnets. This can be accomplished by updating the desired $\mathbf{B}$ in (24) as

$$
\mathbf{B}[k+1]=e^{\mathbb{S}(\omega \Delta t)} \mathbf{B}[k]
$$

where $\Delta t$ is the time step of the control system and the matrix exponential of a skew-symmetric matrix creates a rotation matrix [22]. Thus, the same steady-state rotating control approaches can be performed by an Omnimagnet using (24) and (25) in which, unlike permanent magnets, both the desired field magnitude and orientation are specified.
A rotating magnetic field was used to propel a threaded capsule endoscope mockup down a transparent lumen, which was offset by $120 \mathrm{~mm}$ from the surface of the Omnimagnet, as shown in Fig. 5. Although the trajectory of the capsule is simple (a line), the translating rotational field necessary to drive the capsule uses all $3 \mathrm{DoF}$ available to the Omnimagnet. The desired magnetic field with $\|\mathbf{B}\|=3 \mathrm{mT}$ was updated using (25) for a rotational rate of $4 \pi \mathrm{rad} \cdot \mathrm{s}^{-1}$. The position of the capsule was tracked using a stereo-vision system, although other localization methods, such as the magnetic localization of [20], could be used in the future. This position was used in conjunction with (24) to calculate the currents necessary to produce the desired field at the location of the capsule. The necessary currents were controlled by a dc voltage signal sent from a Sensoray S626 controller card to Advanced Motion Control AMC16A8 current drives at an update rate of $\Delta t=0.01 \mathrm{~s}$

\section{DISCUSSION}

The Omnimagnet prototype developed in this paper uses no form of forced cooling. However, for Omnimagnets to be truly effective, they will need to be cooled. Immersive fluid cooling and forced-convection fluid cooling will enable higher currents, and therefore higher fields, to be generated. An Omnimagnet's dipole strength also increases with size, but this must be balanced against an increase in cost and weight.

By combining multiple Omnimagnets together, it will be possible to create more sophisticated magnetic manipulation systems. For example, the Octomag system uses eight stationary electromagnets to generate $3 \mathrm{DoF}$ force and $2 \mathrm{DoF}$ torque on magnetic devices [7]. A system consisting of three Omnimagnets is essentially nine stationary electromagnets, meaning that similar levels of control as the Octomag seem conceivable. The commercial Stereotaxis Niobe system uses two large orientation-controlled permanent magnets to steer magnetic catheters. A system consisting of two Omnimagnets 
has the ability to recreate the same type of magnetic control. Additionally, the spherical core inside the Omnimagnet makes its use in multi-Omnimagnet systems extremely promising. Because the average magnetization of a spherical core can be solved using only knowledge of the applied field at the center of the sphere, it will be possible to solve for the combined field of multiple Omnimagnets analytically, rather than relying on in situ system calibration.

If an Omnimagnet were constructed with a cubic core, the available ferromagnetic material would increase by $57 \%$, which suggests that the overall strength of the magnet would increase. Using the methodology outlined in this paper, preliminary simulations indicate that the dipole moment of an equivalently sized Omnimagnet with a cubic core would be about $115 \%$ of the no-ferromagnetic-material reference geometry, which is $24 \%$ stronger than the optimal spherical-core geometry. Because the core is no longer spherical, this additional strength is also associated with larger dipole-modeling errors (equivalent to the errors associated with cubic permanent magnets [21]), and a significantly more complicated core magnetization calculation. If multiple Omnimagnets were to be used in concert, the complexity of the mutual magnetization could be prohibitive, requiring in situ calibration. However, if only one Omnimagnet is required for the application, a single Omnimagnet with a cubic core could provide a significant increase in strength with only a marginal increase in reoptimization and development complexity.

\section{CONCLUSION}

The design and optimization of an Omnimagnet was provided. The realized version of the design has an optimal core-radius to outer-length ratio of 0.57 and can achieve field strengths that are $87 \%$ of the unrealizable theoretical reference. The design was optimized to create a dipole-like field with the error relative to the dipole model falling to below $5 \%$ outside of 1.5 minimum-bounding-sphere radii from the center. Manipulation with the Omnimagnet was demonstrated by actuating a helical capsule down a lumen. Not only can the Omnimagnet create a rotating dipole field like a permanent magnet, but it can also control the field strength like a standard electromagnet. This enables new control methodologies to be explored. Moreover, the spherical core will allow the combined field of multiple Omnimagnets to be solved analytically.

\section{APPENDIX}

This appendix provides the derivation of the approximate inductance of a square-cross-sectional solenoid with a spherical core. The magnetic flux $\Phi$ through a current loop is related to the inductance of the loop $\mathcal{L}$ by

$$
\Phi \equiv \int \mathbf{B} \cdot d \mathbf{a}=\mathcal{L} I
$$

where $d \mathbf{a}$ is the differential area of the plane inside the loop [18]. It should be noted that if the Omnimagnet's solenoids are mutually orthogonal, the mutual inductance between each solenoid is zero because of the dot product in (26). Thus, the inductance of a collection of connected loops is

$$
\mathcal{L}=\sum_{i=1}^{N} \frac{\Phi_{i}}{I}
$$

where $N$ is the total number of loops. Letting $\eta$ be the area packing efficiency of the wire, the total number of loops can be obtained geometrically

$$
N=\frac{\eta T L}{A_{w}}=\frac{\eta\left(\beta_{2}-\beta_{1}\right) L^{2}}{2 A_{w}} .
$$

Although possible, computing the sum in (27) is cumbersome. If, instead of summing fluxes and loops in the solenoid, we integrate an effective flux and cross-sectional loop density over the solenoid, the computation of the inductance can be greatly simplified. The loop density, gleaned by inspection of (28), is $\eta / A_{w}$. Thus, in terms of an effective loop density the total number of loops is

$$
N=\int d N=\int_{-L / 2}^{L / 2} \int_{0}^{T} \frac{\eta}{A_{w}} d T d L .
$$

Keeping with the theme of non-dimensionalizing the geometric lengths, we use the change of variable $d T=L d \zeta_{T}$ and $d L=L d \zeta_{L}$. With this substitution we obtain

$$
N=\int_{-1 / 2}^{1 / 2} \int_{0}^{\frac{\left(\beta_{2}-\beta_{1}\right)}{2}} \frac{\eta L^{2}}{A_{w}} d \zeta_{T} d \zeta_{L}
$$

and (27) can be approximated as

$$
\mathcal{L} \approx \frac{\eta L^{2}}{I A_{w}} \int_{-1 / 2}^{1 / 2} \int_{0}^{\frac{\left(\beta_{2}-\beta_{1}\right)}{2}} \Phi\left(\zeta_{T}, \zeta_{L}\right) d \zeta_{T} d \zeta_{L} .
$$

It is now left to define the effective flux density in the solenoid $\Phi\left(\zeta_{T}, \zeta_{L}\right)$. Referring to (26), the flux density is formed by an area component and a field component. The area associated with the loop density can be obtained geometrically and is $L^{2}\left(2 \zeta_{T}+\beta_{1}\right)^{2}$; the loop-density flux is therefore

$$
\Phi\left(\zeta_{T}, \zeta_{L}\right)=L^{2}\left(2 \zeta_{T}+\beta_{1}\right)^{2} \mathcal{B}\left(\zeta_{T}, \zeta_{L}\right) .
$$

The magnetic field $\mathcal{B}\left(\zeta_{T}, \zeta_{L}\right)$ associated with the loop-density flux is the average field within the differential loop, which is difficult to calculate exactly but will be simplified with another approximation.

Without loss of generality, we will take the axis of the solenoid to be oriented in the cartesian $z$ direction. The calculation of $\mathcal{B}\left(\zeta_{T}, \zeta_{L}\right)$ is simplified by assuming the field inside the solenoid when no core is present $\mathcal{B}_{S}(z)$ varies along the solenoid axis but is constant across the cross section

$$
\begin{aligned}
\mathcal{B}_{s}(z)= & \frac{L J \mu_{0}}{\pi} \int_{\beta_{1}}^{\beta_{2}}\left(\operatorname{atan}\left(\frac{1+2 z / L}{\sqrt{(1+2 z / L)^{2}+2 \zeta^{2}}}\right)\right. \\
& \left.+\operatorname{atan}\left(\frac{1-2 z / L}{\sqrt{(1-2 z / L)^{2}+2 \zeta^{2}}}\right)\right) d \zeta .
\end{aligned}
$$


Note that (33) reduces to (11) when $z=0$. Since we are magnetizing the spherical core in its linear region, the field is the superposition of the field from only the solenoid current $\mathcal{B}_{S}\left(L \zeta_{L}\right)$ and the field only from the magnetization of the core $\mathcal{B}_{c}\left(\zeta_{T}, \zeta_{L}\right)$; thus, $\mathcal{B}\left(\zeta_{T}, \zeta_{L}\right) \approx \mathcal{B}_{S}\left(L \zeta_{L}\right)+\mathcal{B}_{c}\left(\zeta_{T}, \zeta_{L}\right)$. With this simplification, the inductance due to the solenoid with a core can be split into two separate problems: the inductance of a solenoid with no core and the inductance due only to the magnetization of the core.

Using (31)-(33), the inductance for a square solenoid with no core is

$$
\begin{aligned}
\mathcal{L}_{s} & \approx \frac{\eta L^{4}}{A_{w} I} \int_{-1 / 2}^{1 / 2} \int_{0}^{\frac{\beta_{2}-\beta_{1}}{2}}\left(\beta_{1}+2 \zeta_{T}\right)^{2} \mathcal{B}_{S}\left(L \zeta_{L}\right) d \zeta_{T} d \zeta_{L} \\
& \approx \frac{\eta L^{4}\left(\beta_{2}^{3}-\beta_{1}^{3}\right)}{6 A_{w} I} \int_{-1 / 2}^{1 / 2} \mathcal{B}_{S}\left(\zeta_{L} L\right) d \zeta_{L} .
\end{aligned}
$$

The inductance contribution of the core is more difficult to calculate; however, the calculation can be split into two parts as well: that which is associated with the uniform field inside the core, and that which is associated with the dipole field outside the core.

Inside of a uniformly magnetized spherical core, the magnetic field due only to the magnetization of the material is uniform and is [18]

$$
\mathcal{B}_{c, i}=\frac{2}{3} \mu_{0}\|\mathbf{M}\|=2 \mathcal{B}_{s}(0) .
$$

When the magnetization is due to an external field, the total internal field is then $2 \mathcal{B}_{s}(0)+\mathcal{B}_{s}(0)=3 \mathcal{B}_{s}(0)$, which is expected since the demagnetization factor of a sphere is $1 / 3$. Since the external field is already accounted for in the solenoid-alone inductance, the field of $2 \mathcal{B}_{c}(0)$ will be used to calculate the part of the inductance only due to magnetizing the core material. The inductance associated with the field inside the core is thus

$$
\begin{aligned}
\mathcal{L}_{c, i} & =\frac{\eta L^{4}\left(\beta_{2}-\beta_{1}\right)}{2 A_{w} I} \int_{-\beta_{c} / 2}^{\beta_{c} / 2} \pi\left(\frac{1}{4} \beta_{c}^{2}+\zeta_{1}^{2}\right) \mathcal{B}_{s}(0) d \zeta \\
& =\frac{\pi \eta L^{4}\left(\beta_{2}-\beta_{1}\right) \beta_{c}^{3} \mathcal{B}_{s}(0)}{6 A_{w} I}
\end{aligned}
$$

where $\beta_{c}$ is being introduced as the ratio of core diameter to solenoid length.

Outside of the magnetized core, the field is purely dipolar with the dipole moment given by (10). To numerically determine the inductance of this field, the differential flux is given by

$$
\frac{d \Phi}{d a}=\frac{\mu_{0}\|\mathbf{m}\|}{4 \pi\|\mathbf{p}\|^{3}} \hat{\mathbf{z}}^{\mathrm{T}}\left(3 \hat{\mathbf{p}} \hat{\mathbf{p}}^{\mathrm{T}}-\mathbb{I}\right) \hat{\mathbf{z}}=\frac{L^{3} \beta_{c}^{3} \mathcal{B}_{s}(0)}{8\|\mathbf{p}\|^{3}}\left(3\left(\hat{\mathbf{z}}^{\mathrm{T}} \hat{\mathbf{p}}\right)^{2}-1\right) .
$$

The inductance is given by (31) where the flux is the flux outside of the core and inside of the loop-density width being integrated

$$
\begin{aligned}
\mathcal{L}_{c, \text { dipole }} & =\frac{\eta \beta_{c}^{3} L^{4}}{8 A_{w} I} \mathcal{B}(0) \\
& \iiint \int\left(\frac{2\left(\zeta_{L} L\right)^{2}-x^{2}-y^{2}}{\left(x^{2}+y^{2}+\left(\zeta_{L} L\right)^{2}\right)^{5 / 2}}\right) d x d y d \zeta_{T} d \zeta_{L} .
\end{aligned}
$$

Numerical analysis of this contribution indicates it accounts for less than $2 \%$ of the total inductance and will be neglected for simplicity.

Therefore, the total inductance is the combination of the inductance due to the solenoid alone, plus the inductance due to the uniform field inside of the magnetized core

$$
\begin{aligned}
\mathcal{L} \approx & \frac{\eta L^{4}}{6 A_{w} I}\left(\beta_{2}^{3}-\beta_{1}^{3}\right) \int_{-1 / 2}^{1 / 2} \mathcal{B}_{s}\left(\zeta_{2} L\right) d \zeta_{2} \\
& +\frac{\eta L^{4}}{6 A_{w} I} \pi\left(\beta_{2}-\beta_{1}\right) \beta_{c}^{3} \mathcal{B}_{s}(0) .
\end{aligned}
$$

Combining (33) and (39), this inductance can be rewritten as a function of relative solenoid shape scaled by a function of solenoid size, as shown in (21), where $f_{1}(\cdot)$ is the integration described in (39) with $f_{2}(\cdot)$ comprising the integrand of (33).

\section{ACKNOWLEDGMENT}

This work was supported by the National Science Foundation under Grant 0952718 and Grant 0654414.

\section{REFERENCES}

[1] T. Honda, K. I. Arai, and K. Ishiyama, "Micro swimming mechanisms propelled by external magnetic fields," IEEE Trans. Magn., vol. 32, no. 5, pp. 5085-5087, Sep. 1996.

[2] D. Meeker, E. H. Maslen, R. C. Ritter, and F. Creighton, "Optimal realization of arbitrary forces in a magnetic stereotaxis system," IEEE Trans. Magn., vol. 32, no. 2, pp. 320-328, Mar. 1996.

[3] M. Sendoh, K. Ishiyama, and K. Arai, "Direction and individual control of magnetic micromachine," IEEE Trans. Magn., vol. 38, no. 5, pp. 3356-3358, Sep. 2002.

[4] H. Lee, A. Purdon, and R. M. Westervelt, "Micromanipulation of biological systems with microelectromagnets," IEEE Trans. Magn., vol. 40, no. 4, pp. 2991-2993, Aug. 2004.

[5] S. Martel, C. C. Tremblay, S. Ngakeng, and G. Langlois, "Controlled manipulation and actuation of micro-objects with magnetotactic bacteria," Appl. Phys. Lett., vol. 89, pp. 233904-1-233904-3, Dec. 2006.

[6] S. Martel, J.-B. Mathieu, O. Felfoul, A. Chanu, E. Aboussouan, S. Tamaz, et al., "Automatic navigation of an untethered device in the artery of a living animal using a conventional clinical magnetic resonance imaging system," Appl. Phys. Lett., vol. 90, pp. 114105-1-114105-3, Mar. 2007.

[7] M. P. Kummer, J. J. Abbott, B. E. Kratochvil, R. Borer, A. Sengul, and B. J. Nelson, "Octomag: An electromagnetic system for 5-DOF wireless micromanipulation," IEEE Trans. Robot., vol. 26, no. 6, pp. 1006-1017, Dec. 2010.

[8] M. Mehrtash, N. Tsuda, and M. Khamesee, "Bilateral macro-micro teleoperation using magnetic levitation," IEEE/ASME Trans. Mechatron., vol. 16, no. 3, pp. 459-469, Jun. 2011.

[9] M. H. Hagiwara, T. K. Kawahara, Y. Yamanishi, and F. Arai, "Driving method of microtool by horizontally arranged permanent magnets for single cell manipulation," Appl. Phys. Lett., vol. 97, pp. 013701-1-013701-3, Jul. 2010.

[10] G. Ciuti, P. Valdastri, A. Menciassi, and P. Dario, "Robotic magnetic steering and locomotion of capsule endoscope for diagnostic and surgical endoluminal procedures," Robotica, vol. 28, no. 2, pp. 199-207, Mar. 2010.

[11] S. Yim and M. Sitti, "Design and rolling locomotion of a magnetically actuated soft capsule endoscope," IEEE Trans. Robot., vol. 28, no. 1, pp. 183-194, Feb. 2012. 
[12] A. W. Mahoney, D. L. Cowan, K. M. Miller, and J. J. Abbott, "Control of untethered magnetically actuated tools using a rotating permanent magnet in any position," in Proc. 4th IEEE RAS EMBS Int. Conf. Robot. Autom., Jun. 2012, pp. 1632-1637.

[13] S. Ernst, F. Ouyang, C. Linder, K. Hertting, F. Stahl, J. Chun, et al., "Initial experience with remote catheter ablation using a novel magnetic navigation system magnetic remote catheter ablation," Circulation, vol. 109, no. 12 , pp. 1472-1475, Mar. 2004

[14] A. W. Mahoney and J. J. Abbott, "Managing magnetic force applied to a magnetic device by a rotating dipole field," Appl. Phys. Lett., vol. 99, no. 13, pp. 134103-1-134103-3, Sep. 2011.

[15] F. Raab, E. Blood, T. Steiner, and H. Jones, "Magnetic position and orientation tracking system," IEEE Trans. Aerosp. Electron. Syst., vol. 15, no. 5, pp. 709-718, Sep. 1979.

[16] R. Carta, J. Thoné, and R. Puers, "A wireless power supply system for robotic capsular endoscopes," Sens. Actuators A, Phys., vol. 162, no. 2, pp. 177-183, Aug. 2010.
[17] A. J. Petruska and J. J. Abbott, "An omnidirectional electromagnet for remote manipulation," in Proc. IEEE ICRA, May 2013, pp. 814-819.

[18] D. J. Griffiths, Introduction to Electrodynamics. Englewood Cliffs, NJ, USA: Prentice-Hall, 1999.

[19] A. W. Mahoney, S. E. Wright, and J. J. Abbott, "Managing the attractive magnetic force between an untethered magnetically actuated tool and a rotating permanent magnet," in Proc. IEEE ICRA, May 2013, pp. 5346-5351.

[20] K. M. Popek, A. W. Mahoney, and J. J. Abbott, "Localization method for a magnetic capsule endoscope propelled by a rotating magnetic dipole field," in Proc. IEEE ICRA, May 2013, pp. 5328-5333.

[21] A. J. Petruska and J. J. Abbott, "Optimal permanent-magnet geometries for dipole field approximation," IEEE Trans. Magn., vol. 49, no. 2, pp. 811-819, Feb. 2013.

[22] R. M. Murray, Z. Li, and S. S. Sastry, A Mathematical Introduction to Robotic Manipulation. Boca Raton, FL, USA: CRC, 1994. 